\title{
OUTCOME OF PATIENTS WITH SPONTANEOUS INTRACEREBRAL HEMORRHAGE AT TERTIARY CARE HOSPITAL IN NEPAL.
}

\section{Dilli Ram Kafle}

\begin{abstract}
:
Nontraumatic intracerebral hemorrhage is bleeding into the parenchyma of the brain that may extend into the ventricles and, in rare cases, the subarachnoid space. Intracerebral hemorrhage accounts for 10 to 15 percent of all cases of stroke and is associated with the highest mortality rate, with only 38 percent of affected patients surviving the first year. It is a prospective analytical study carried out at Tribhuvan Universiy Teaching Hosital between April 2012 and April 2013. A total of 100 patients were admitted to the medical ward and intensive care unit during this period. Patients were followed until they were discharged from the hospital. Spontaneous Intracerebral bleeding was found to be more common in males than women. 85 percent of patients were above the age of 50. 45 Percent of the patients had onset in the early morning between 5AM and 11AM.Patients diagnosed with hypertension but not taking antihypertensive medications or taking them irregularly were more likely to have the vascular event than those who were taking the medication regularly. Patients also had very high blood pressure at the time of presentation. 46 percent of the patients had putaminal bleeding. 44 percent of patients were discharged before 10 days. Only 8 percent of patients had a stay longer than 30 days. 8 percent of the patients needed mechanical ventilation during their hospital stay .The case fatality rate was 6 percent.
\end{abstract}

Keyword: Intracerebral hemorrhage, case fatality, hematoma

\section{Introduction}

Nontraumatic intracerebral hemorrhage is bleeding into the parenchyma of the brain that may extend into the ventricles and, in rare cases, the subarachnoid space. Intracerebral hemorrhage accounts for 10 to 15 percent of all cases of stroke and is associated with the highest mortality rate, with only 38 percent of affected patients surviving the first year. ${ }^{1}$ Depending on the underlying cause of bleeding; intracerebral hemorrhage is classified as either primary or secondary. Primary intracerebral hemorrhage, accounting for 78 to 88 percent of cases, originates from the spontaneous rupture of small vessels damaged by chronic hypertension or amyloid angiopathy. $^{2}$ Secondary intracerebral hemorrhage occurs in a minority of patients in association with vascular abnormalities (such as arteriovenous malformations and aneurysms), tumors, or impaired coagulation. Although hypertensive intracerebral hemorrhage remains the most common form of intracerebral hemorrhage, underlying vascular abnormalities should always be considered in appropriate circumstances because of the high risk of recurrent hemorrhage and available treatment options. ${ }^{3-9}$

\section{Epidemio ogic Features}

\section{Incidence:}

The worldwide incidence of intracerebral hemorrhage ranges from 10 to 20 cases per 
100,000 population ${ }^{10,11}$ and increases with age. $^{10,12}$ Intracerebral hemorrhage is more common in men than women, particularly those older than 55 years of age, ${ }^{12,13}$ and in certain populations, including blacks and Japanese. ${ }^{10,14}$

\section{Risk Factors}

Hypertension is the most important risk factor for spontaneous intracerebral hemorrhage. ${ }^{15}$ Hypertension increases the risk of intracerebral hemorrhage, particularly in persons who are not compliant with antihypertensive medication, are 55 years of age or younger, or are smokers. ${ }^{16,17}$ Improved control of hypertension appears to reduce the incidence of intracerebral hemorrhage. In the Hypertension Detection and Follow-up Program, persons with hypertension (defined as a diastolic blood pressure of at least $95 \mathrm{~mm}$ $\mathrm{Hg}$ ) who were 30 to 69 years of age and who received standardized antihypertensive therapy had a risk of stroke (including intracerebral hemorrhage) of 1.9 per 100 persons, as compared with a risk of 2.9 per 100 persons in those who received routine community care. ${ }^{18}$ This approach was associated with an absolute reduction in risk of 46 percent in persons who were 65 years of age or older. In the Systolic Hypertension in the Elderly Program, the five-year incidence of all strokes, including intracerebral hemorrhage, in patients older than 60 years who had a systolic blood pressure of at least $160 \mathrm{~mm} \mathrm{Hg}$ was 5.2 per 100 with antihypertensive treatment and 8.2 per 100 with placebo treatment. ${ }^{19}$

Excessive use of alcohol also increases the risk of intracerebral hemorrhage by impairing coagulation and directly affecting the integrity of cerebral vessels. ${ }^{20,21}$ Other less well established risk factors include serum cholesterol levels of less than $160 \mathrm{mg}$ per deciliter (4.1 mmol per liter), particularly among patients with hypertension, and genetic factors such as mutations in genes encoding the $\mathbf{\square}$ subunit of factor XIII (which is involved in the formation of cross-linked fibrin). ${ }^{22-24}$ Cerebral amyloid angiopathy, which is characterized by the deposition of $\mathbf{0}$ amyloid protein in the blood vessels of the cerebral cortex and leptomeninges, is another risk factor for intracerebral hemorrhage, particularly in elderly persons Surgical Specimens from the Matrix of a Hematoma Associated with Amyloid Angiopathy.). O'Donnell et al.reported that the presence of the $\mathbf{\square} 2$ and $\mathbf{\square} 4$ alleles of the apolipoprotein $E$ gene was associated with a tripling of the risk of recurrent hemorrhage among survivors of lobar intracerebral hemorrhage related to amyloid angiopathy. These alleles are associated with increased deposition of $\mathbf{0}$ amyloid protein and degenerative changes (such as fibrinoid necrosis) in the vessel wall, respectively. The expression of either allele appears to increase the risk of intracerebral hemorrhage by augmenting the vasculopathic effects of amyloid deposition in cerebral vessels.

\section{Pathophysio ogica Features}

\section{Patho ogic Process}

Intracerebral hemorrhages commonly occur in the cerebral lobes, basal ganglia, thalamus, brain stem (predominantly the pons), and cerebellum ${ }^{25}$ Extension into the ventricles occurs in association with deep, large hematomas. Edematous parenchyma, often discolored by degradation products of hemoglobin, is visible adjacent to the clot. ${ }^{26}$ Histologic sections are characterized by the presence of edema, neuronal damage, macrophages, and neutrophils in the region surrounding the hematoma. The hemorrhage spreads between planes of white-matter cleavage with minimal destruction, leaving nests of intact neural tissue within and 
surrounding the hematoma. ${ }^{25,26}$ This pattern of spread accounts for the presence of viable and salvageable neural tissue in the immediate vicinity of the hematoma.

\section{Origin of Hematoma}

Intraparenchymal bleeding results from the rupture of the small penetrating arteries that originate from basilar arteries or the anterior, middle, or posterior cerebral arteries. Degenerative changes in the vessel wall induced by chronic hypertension reduce compliance and increase the likelihood of spontaneous rupture. In 1868, Charcot and Bouchard attributed bleeding to rupture at points of dilatation in the walls of small arterioles (microaneurysms).27-29 These morphologic entities were later found to be subadventitial hemorrhages or extravascular clots resulting from endothelial damage by the hematoma. ${ }^{29} \quad$ Electron-microscopical studies suggest that most bleeding occurs at or near the bifurcation of affected arteries, where prominent degeneration of the media and smooth muscles can be seen. ${ }^{29}$

\section{Progression of Hematoma}

Initially, intracerebral hemorrhage was considered to be a monophasic event that stopped quickly as a result of clotting and tamponade by the surrounding regions. This impression is incorrect, as demonstrated by computed tomographic (CT) scans showing that hematomas expand over time

.$^{30,31}$ In a study of 103 patients, Brott et al. found that the hematoma expanded in 26 percent of the patients within 1 hour after the initial CT scan and in another 12 percent within 20 hours. Kazui et al.reported that the hematoma expanded in 41 of 204 patients (20 percent) with intracerebral hemorrhage, occurring in 36 percent of patients who presented within three hours after the onset of the hemorrhage and in 11 percent of those who presented more than three hours after the onset. This expansion has been attributed to continued bleeding from the primary source and to the mechanical disruption of surrounding vessels. Acute hypertension, a local coagulation deficit, or both ${ }^{32}$ may be associated with expansion of the hematoma. ${ }^{33,34}$

\section{Secondary Neurona Injury after Intracerebra Hemorrhage}

The presence of hematoma initiates edema and neuronal damage in surrounding parenchyma. Fluid begins to collect immediately in the region around the hematoma, and edema usually persists for up to five days, ${ }^{35}$ although it has been observed for as long as two weeks after a stroke. ${ }^{36}$ Early edema around the hematoma results from the release and accumulation of osmotically active serum proteins from the clot. ${ }^{37}$ Vasogenic edema and cytotoxic edema subsequently follow owing to the disruption of the blood-brain barrier, the failure of the sodium pump, and the death of neurons. ${ }^{38,39}$

The delay in the breakdown of the bloodbrain barrier and the development of cerebral edema after intracerebral hemorrhage suggest that there may be secondary mediators of both neural injury and edema. It is currently thought that blood and plasma products mediate most secondary processes that are initiated after an intracerebral hemorrhage. ${ }^{40-46}$ Neuronal death in the region around the hematoma is predominantly necrotic, with recent evidence suggesting the presence of programmed cell death (apoptosis) associated with nuclear factor- $\mathrm{B}$ expression in neuralcell nuclei. ${ }^{46}$

\section{C inica Features}

\section{Neuro ogic Status at Presentation}

Patients with a large hematoma usually have a decreased level of consciousness ${ }^{47}$ as a result of increased intracranial pressure and the 
direct compression or distortion of the thalamic and brain-stem reticular activating system. ${ }^{48}$ Decreased central benzodiazepinereceptor binding on cortical neurons in the presence of small, deep lesions may also contribute to altered consciousness. ${ }^{49}$ Patients with a supratentorial intracerebral hemorrhage involving the putamen, caudate, and thalamus have contralateral sensory-motor deficits of varying severity owing to the involvement of the internal capsule. Abnormalities indicating higher-level cortical dysfunction, including aphasia, neglect, gaze deviation, and hemianopia, may occur as a result of the disruption of connecting fibers in the subcortical white matter and functional suppression of overlying cortex, known as diaschisis. ${ }^{50}$

In patients with an infratentorial intracerebral hemorrhage, signs of brain-stem dysfunction include abnormalities of gaze, cranial-nerve abnormalities, and contralateral motor deficits. ${ }^{51}$ Ataxia, nystagmus, and dysmetria are prominent when the intracerebral hemorrhage involves the cerebellum. Common nonspecific symptoms include headache and vomiting due to increased intracranial pressure and meningismus resulting from blood in the ventricles. ${ }^{52,53}$

\section{Secondary Deterioration}

In one fourth of patients with intracerebral hemorrhage who are initially alert, a deterioration in the level of consciousness occurs within the first 24 hours after onset of the hemorrhage. ${ }^{54,55}$ The presence of a large hematoma and ventricular blood increases the risk of subsequent deterioration and death. Expansion of the hematoma is the most common cause of underlying neurologic deterioration within the first three hours after the onset of hemorrhage. Worsening cerebral edema is also implicated in neurologic deterioration that occurs within 24 to 48 hours after the onset of hemorrhage. ${ }^{55}$ infrequently; late deterioration is associated with progression of edema during the second and third weeks after the onset.

The mortality rate six months after spontaneous intracerebral hemorrhage ranges from 23 to 58 percent. $^{56-58}$ A low score on the Glasgow Coma Scale, a large volume of the hematoma, and the presence of ventricular blood on the initial CT scan are factors that have been consistently identified as predictive of a high mortality rate Broderick et al. found that the mortality rate at one month was best predicted by determining the initial score on the Glasgow Coma Scale and the initial volume of the hematoma. In their study, patients who initially had a score of less than 9 on the Glasgow Coma Scale and a hematoma volume of more than $60 \mathrm{ml}$ had a mortality rate of 90 percent at one month, whereas patients with a score of 9 or greater and a hematoma volume of less than $30 \mathrm{ml}$ had a mortality rate of 17 percent.

A rapid manual method to measure the volume of the hematoma on CT scans has been developed and validated. In this method, the estimated volume of the hematoma is half the product of $\mathrm{A}, \mathrm{B}$, and $\mathrm{C}$, where $\mathrm{A}$ is the greatest diameter of the hemorrhage on the CT scan, B is the diameter perpendicular to $\mathrm{A}$, and $\mathrm{C}$ is the number of slices showing hematoma multiplied by the slice thickness. ${ }^{56}$

Although the rapid onset of abnormalities and a decreased level of consciousness suggest the diagnosis of intracerebral hemorrhage distinguishing definitively between cerebral infarction and intracerebral hemorrhage requires imaging of the brain. ${ }^{59}$ On the initial CT scan, the location and size of the hematoma, the presence of ventricular blood, and the occurrence of hydrocephalus should be noted. Selected patients should undergo conventional angiography to look for secondary causes of intracerebral hemorrhage, such as aneurysms, arteriovenous 
malformations, and vasculitis. Zhu et al. ${ }^{60}$ reported abnormalities on angiography in 49 percent of patients with lobar hemorrhage and 65 percent of patients with isolated intraventricular hemorrhage. These authors also reported that 48 percent of the patients who were normotensive and 45 years of age or younger had abnormalities on angiography, whereas hypertensive patients who were older than 45 years of age had no underlying vascular abnormalities.

On the basis of this evidence, patients with lobar or primary intraventricular hemorrhage should undergo angiography regardless of age or the presence or absence of a history of hypertension. Patients with putaminal, thalamic, or cerebellar hemorrhage should undergo conventional angiography if they are normotensive and 45 years of age or younger. The guidelines of the American Heart Association2 recommend angiography for all patients with no clear cause of hemorrhage who are candidates for surgery, particularly young patients without hypertension whose condition is clinically stable. The timing of conventional angiography depends on the patient's clinical condition and the urgency of surgery. Magnetic resonance imaging with gadolinium as a contrast medium and magnetic resonance angiography can also be used to identify secondary causes of intracerebral hemorrhage, although their sensitivity is not well established. ${ }^{61}$ Conventional angiography should also be considered in patients who have subarachnoid blood associated with a parenchymal clot and in patients who have recurrent hemorrhages. In patients who have initially negative findings on imaging but who have a high likelihood of secondary intracerebral hemorrhage on the basis of clinical findings, angiography should perhaps be repeated two to four weeks after the resolution of the hematoma, when vascular anomalies may become visible.

\section{Methodo ogy:}

It was a prospective analytical study carried out at Tribhuvan University Teaching Hospital. All the patients admitted at medical ward and intensive care unit from April 2012 to April 2013 were followed from the time of admission until they were discharged. Their clinical characteristics including vitals and outcome with regard to presenting level of consciousness and neuro-imaging was recorded

\section{Resu t}

From April 2012 to April 2013 a total of 100 patients with spontaneous intracerebral bleeding were admitted at Tribhuvan University Teaching Hospital medical ward and intensive care unit. There were 63 males and 27 females.

Age distribution of patient's presentation with spontaneous intracerebral bleeding was as follows. Less than 20 years of age: 1 patient. 20-29: 1 Patient. 30-39: 2 patients. 40-49: 11 Patients. 50-59: 19 patients. 60-69: 29 Patients. 70-79: 20 patients. Above 80 years: 17 patients

Blood Pressure at the time of admission: 32percent of patients had blood pressure more than 180/110 mmhg.28 percent had BP more than 160/100 mmhg, 6 percent had BP of more than 140/90 mmhg. Rest of the patients (34\%) had normal BP at the time of admission. 20 percent of the patients who were diagnosed with blood pressure were taking antihypertensive medication regularly. 36 percent of patients who were diagnosed with hypertension were either not taking antihypertensive medicine or taking them irregularly .The latter group were not taking the medication at least few days before the vascular event. The remaining $44 \%$ of patients did not have a history of hypertension. 
Nine percent of patients had a history of diabetes mellitus. 21 percent of patients were smokers.20 percent of patients consumed excessive amount of alcohol

The most common clinical features were hemiparesis and upper neuron type of facial palsy. Vomiting was present in $45 \%$ of patients. The other complaints were headache (43\%), Loss of Consciousness:(17\%), Seizure $(13 \%)$

Time of vascular event: Most of the events (45\%) occurred in the morning between 5 AM and $11 \mathrm{AM}$. The onset was least common in the afternoon between $12 \mathrm{pm}$ to $6 \mathrm{pm}$.

Glassgow Coma Scale at presentation: The majority of patients $(66 \%)$ admitted to the hospital had a Glassgow coma scale of 15 .

Neuroimaging finding:

CT scan was done in all the patients at the time of admission. $46 \%$ of patients had primarily putaminal bleeding.Lobar bleeding was present in 28\% of patients. Thalamic bleeding in 13\%.Cerebellar bleeding in 3\% and Pontine bleeding in $4 \%$ of patients.Intraventricular extension was present in $26 \%$ of patients.

Duration of hospital stay: $44 \%$ of patients were discharged from the hospital before 10 days. Only $8 \%$ of Patients had a stay more than 30 days.

Outcome: $90 \%$ of the patients who were admitted improved and were discharged from the hospital. The mortality rate was $6 \%$. Condition of $4 \%$ patients deteriorated and were taken to home on family members request.8\%of patients needed mechanical ventilation. $5 \%$ of patients had surgical evacuation of their brain hematoma

\section{Discussion}

In my study it was found that hemorrhagic stroke was more common in male than female. The incidence of hemorrhagic stroke increased dramatically after the age of 50.The majority of events occurred in the early morning. Giroud M, Gras P, Chadan N, et al. also found hemorrhagic stroke to be more common in males above age $55 .^{12}$

Putaminal bleeding was the most common. Almost a quarter of patients had intraventricular extension. Equal number of bleeding occurred in those who were diagnosed with hypertension and taking medications regularly and those who were taking them irregularly or not taking the medication at all. Hypertension is the most important risk factor for spontaneous intracerebral hemorrhage. ${ }^{15}$ Hypertension increases the risk of intracerebral hemorrhage, particularly in persons who are not compliant with antihypertensive medication, are 55 years of age or younger, or are smokers. ${ }^{16,17}$

Most patients had very high blood pressure at the time of presentation. Majority of patients had favorable outcome in terms of mortality. In my study the mortality rate was 6 percent when the patients were followed until their discharge. 4 percent had their condition deteriorated and were taken home by their family members. Glassgow coma score less than 9 and intraventricular extension was predictor of poor prognosis and mortality. Broderick et al. ${ }^{56}$ found that the mortality rate at one month was best predicted by determining the initial score on the Glasgow Coma Scale and the initial volume of the hematoma. In their study, patients who initially had a score of less than 9 on the Glasgow Coma Scale and a hematoma volume of more than $60 \mathrm{ml}$ had a mortality rate of 90 percent at one month, whereas patients with a score of 9 or greater and a hematoma volume of less than $30 \mathrm{ml}$ had a mortality rate of 17 percent.

\section{Conc usion:}

Spontaneous intracerebral hemorrhage is more common among those hypertensive 
patients who are not taking medication regularly. The onset is most common in the early morning. Majority of patients have very high blood pressure at presentation. Low Glassgow coma scale and intraventricular extension predicted poor outcome among those patient.

\section{References}

1. Dennis MS, Burn JP, Sandercock PA, Bamford JM, Wade DT, War ow CP. Long-term survival after first-ever stroke: the Oxfordshire Community Stroke Project. Stroke 1993;24:796-800

2. Fou kes MA, Wo f PA, Price TR, Mohr JP, Hier DB. The Stroke Data Bank: design, methods, and baseline characteristics. Stroke 1988;19:547554

3. Arakawa S, Saku Y, Ibayashi S, Nagao T, Fujishima M. Blood pressure control and recurrence of hypertensive brain hemorrhage. Stroke 1998;29:1806-1809

4. O'Donne HC, Rosand J, Knudsen KA, et a . Apolipoprotein E genotype and the risk of recurrent lobar intracerebral hemorrhage. $\mathrm{N}$ Engl J Med 2000;342:240-245

5. The Arteriovenous Malformation Study Group. Arteriovenous malformations of the brain in adults. N Engl J Med 1999;340:1812-1818

6. Jane JA, Kasse NF, Torner JC, Winn HR. The natural history of aneurysms and arteriovenous malformations. J Neurosurg 1985;62:321-323

7. Kondzio ka D, Lunsford LD, Kest e JR. The natural history of cerebral cavernous malformations. J Neurosurg 1995;83:820-824

8. Naff NJ, Wemmer J, Hoenig-Rigamonti $\mathbf{K}$, Rigamonti DR. A longitudinal study of patients with venous malformations: documentation of a negligible hemorrhage risk and benign natural history. Neurology 1998;50:1709-1714

9. Preter M, Tzourio C, Ameri A, Bousser MG. Long-term prognosis in cerebral venous thrombosis: follow-up of 77 patients. Stroke 1996;27:243-246

10. Broderick JP, Brott T, Tomsick T, Huster G, Mi er R. The risk of subarachnoid and intracerebral hemorrhages in blacks as compared with whites. N Engl J Med 1992;326:733-736
11. Fur an AJ, Whisnant JP, E veback LR. The decreasing incidence of primary intracerebral hemorrhage: a population study. Ann Neurol $1979 ; 5: 367-373$

12. Giroud M, Gras P, Chadan N, et a . Cerebral hemorrhage in a French prospective population study. J Neurol Neurosurg Psychiatry 1991;54:595-598

13. Sacco RL, Mayer SA. Epidemiology of intracerebral hemorrhage. In: Feldmann E, ed. Intracerebral hemorrhage. Armonk, N.Y.: Futura Publishing, 1994:3-23.

14. Suzuki K, Kutsuzawa T, Takita K, et a . Clinico-epidemiologic study of stroke in Akita, Japan. Stroke 1987;18:402-406

15. Brott $\mathbf{T}$, Tha inger $\mathbf{K}$, Hertzberg $\mathbf{V}$. Hypertension as a risk factor for spontaneous intracerebral hemorrhage. Stroke 1986;17:10781083

16. Thrift AG, McNei JJ, Forbes A, Donnan GA. Three important subgroups of hypertensive persons at greater risk of intracerebral hemorrhage. Hypertension 1998;31:1223-1229

17. Qureshi AI, Suri MAK, Safdar K, Otten ips JR, Janssen RS, Franke MR. Intracerebral hemorrhage in blacks: risk factors, subtypes, and outcome. Stroke 1997;28:961-964

18. Hypertension Detection and Follow-up Program Cooperative Group. Five-year findings of the Hypertension Detection and Follow-up Program. III. Reduction in stroke incidence among persons with high blood pressure. JAMA 1982;247:633638

19. SHEP Cooperative Research Group. Prevention of stroke by antihypertensive drug treatment in older persons with isolated systolic hypertension: final results of the Systolic Hypertension in the Elderly Program (SHEP). JAMA 1991;265:3255-3264

20. K atsky AL, Armstrong MA, Friedman GD. Alcohol use and subsequent cerebrovascular disease hospitalizations. Stroke 1989;20:741-746

21. Gore ick PB. Alcohol and stroke. Stroke 1987; 18:268-271

22. Iso H, Jacobs DR Jr, Wentworth D, Neaton JD, Cohen JD. Serum cholesterol levels and six-year mortality from stroke in 350,977 men screened for Multiple Risk Factor Intervention Trial. N Engl J Med 1989;320:904-910

23. A berts MJ, Davis JP, Graffagnino C, et a . Endoglin gene polymorphism as a risk factor for 
sporadic intracerebral hemorrhage. Ann Neurol 1997;41:683-686

24. Catto AJ, Koh er HP, Bannan S, Stick and M, Carter A, Grant PJ. Factor XIII Val 34 Leu: a novel association with primary intracerebral hemorrhage. Stroke 1998;29:813-816

25. Mut u N, Berry RG, A pers BJ. Massive cerebral hemorrhage: clinical and pathological correlations. Arch Neurol 1963;8:644-661

26. Morris JH. The nervous system: In: Cotran RS, Kumar V, Robbin SL, eds. Pathologic basis of disease. 3rd ed. Philadelphia: W.B. Saunders, 1999:1385-450.

27. Co e FM, Yates PO. Pseudo-aneurysms in relationship to massive cerebral hemorrhage. J Neurol Neurosurg Psychiatry 1967;30:61-66

28. Takebayashi S, Kaneko M. Electron microscopic studies of ruptured arteries in hypertensive intracerebral hemorrhage. Stroke 1984;14:28-36

29. Fisher CM. Pathological observations in hypertensive cerebral hemorrhage. J Neuropathol Exp Neurol 1971;30:536-550

30. Brott T, Broderick J, Kothari R, et a . Early hemorrhage growth in patients with intracerebral hemorrhage. Stroke 1997;28:1-5

31. Kazui S, Naritomi H, Yamamoto H, Sawada T, Yamaguchi T. Enlargement of spontaneous intracerebral hemorrhage: incidence and time course. Stroke 1996;27:1783-1787

32. O son JD. Mechanisms of homeostasis: effect on intracerebral hemorrhage. Stroke 1993;24:Suppl:I109-I114

33. Broderick JP, Brott TG, Tomsick T, Barsan W, Spi ker J. Ultra-early evaluation of intracerebral hemorrhage. J Neurosurg 1990;72:195-199

34. Kazui S, Minematsu K, Yamamoto H, Sawada T, Yamaguchi T. Predisposing factors to enlargement of spontaneous intracerebral hematoma. Stroke 1997;28:2370-2375

35. Yang GY, Betz AL, Chenevert TL, Brunberg JA, Hoff JT. Experimental intracerebral hemorrhage: relationship between brain edema, blood flow, and blood-brain barrier permeability in rats. J Neurosurg 1994;81:93-102

36. Zazu ia AR, Diringer MN, Derdeyn CP, Powers WJ. Progression of mass effect after intracerebral hemorrhage. Stroke 1999;30:1167-1173

37. Wagner KR, Xi G, Hua Y, et a . Lobar intracerebral hemorrhage model in pigs: rapid edema development in perihematomal white matter. Stroke 1996;27:490-497

38. Mu -Bryce S, Kroh FO, White J, Rosenberg GA. Brain lactate and $\mathrm{pH}$ dissociation in edema: $1 \mathrm{H}-$ and 31P-NMR in collagenase-induced hemorrhage in rats. Am J Physiol 1993;265:R697R702

39. Wagner KR, Xi G, Hau Y, K einho z M, de Courten-Myers GM, Myers RE. Early metabolic alterations in edematous perihematomal brain regions following experimental intracerebral hemorrhage. J Neurosurg 1998;88:1058-1065

40. Lee KR, Co on GP, Betz AL, Keep RF, Kim S, Hoff JT. Edema from intracerebral hemorrhage: the role of thrombin. J Neurosurg 1996;84:91-96

41. Lee KR, Kawai N, Kim S, Sagher O, Hoff JT. Mechanisms of edema formation after intracerebral hemorrhage: effects of thrombin on cerebral blood flow, blood-brain barrier permeability, and cell survival in a rat model. $\mathrm{J}$ Neurosurg 1997;86:272-278

42. Xi G, Wagner KR, Keep FR, et a . Role of blood clot formation on early edema development after experimental intracerebral hemorrhage. Stroke 1998;29:2580-2586

43. Kanno T, Nagata J, Nonomura $\mathbf{K}$, et a . New approaches in the treatment of hypertensive intracerebral hemorrhage. Stroke 1993;24: Suppl:I96-I100

44. Rosenberg GA, Navrati M. Metalloproteinase inhibition blocks edema in intracerebral hemorrhage in the rat. Neurology 1997;48:921-926

45. Kane PJ, Modha P, Strachan RD, et a. The effect of immunosuppression on the of cerebral oedema in an experimental model of intracerebral hemorrhage: whole body and regional irradiation. J Neurol Neurosurg Psychiatry 1992;55:781-786

46. Hickenbottom SL, Grotta JC, Strong R, Denner LA, Aronowski J. Nuclear factor-kappaB and cell death after experimental intracerebral hemorrhage in rats. Stroke 1999;30:2472-2477

47. Mohr JP, Cap an LR, Me ski JW, et a The Harvard Cooperative Stroke Registry: a prospective registry. Neurology 1978;28:754-762

48. Andrews BT, Chi es BW, O sen WL, Pitts LH. The effect of intracerebral hematoma location on the risk of brain-stem compression and on clinical outcome. J Neurosurg 1988;69:518-522

49. Hatazawa J, Shimosegawa E, Satoh T, Kanno I, Uemura K. Central benzodiazepine receptor 
distribution after subcortical hemorrhage evaluated by means of [123]iomazenil and SPECT. Stroke 1995;26:2267-2271

50. Tanaka A, Yoshinaga S, Nakayama Y, Kimura M, Tomonaga M. Cerebral blood flow and clinical outcome in patients with thalamic hemorrhages: a comparison with putaminal hemorrhages. J Neurol Sci 1996;144:191-197

51. Ott KH, Kase CS, Ojemann RG, Mohr JP. Cerebellar hemorrhage: diagnosis and treatment: a review of 56 cases. Arch Neurol 1974;31:160-167

52. Ropper AH, Gress DR. Computerized tomography and clinical features of large cerebral hemorrhages. Cerebrovasc Dis 1991;1:38-42

53. Me o TP, Pinto AN, Ferro JM. Headache in intracerebral hematomas. Neurology 1996;47:494500

54. Qureshi AI, Safdar K, Wei J, et a . Predictors of early deterioration and mortality in black Americans with spontaneous intracerebral hemorrhage. Stroke 1995;26:1764-1767

55. Mayer SA, Sacco RL, Shi T, Mohr JP. Neurologic deterioration in noncomatose patients with supratentorial intracerebral hemorrhage. Neurology 1994;44:1379-1384
56. Broderick JP, Brott TG, Du dner JE, Tomsick T, Huster G. Volume of intracerebral hemorrhage: a powerful and easy-to-use predictor of 30-day mortality. Stroke 1993;24:987-993

57. Lisk DR, Pasteur W, Rhoades H, Putnam RD, Grotta JC. Early presentation of hemispheric intracerebral hemorrhage: prediction of outcome and guidelines for treatment allocation. Neurology 1994;44:133-139

58. Tuhrim S, Horowitz DR, Sacher M, Godbo d JH. Validation and comparison of models predicting survival following intracerebral hemorrhage. Crit Care Med 1995;23:950-954

59. Kim JS, Lee JH, Lee MC. Small primary intracerebral hemorrhage: clinical presentation of 28 cases. Stroke 1994;25:1500-1506[Erratum, Stroke 1994;25:2098.]

60. Zhu XL, Chan MSY, Poon WS. Spontaneous intracranial hemorrhage: which patients need diagnostic cerebral angiography? A prospective study of 206 cases and review of the literature. Stroke 1997;28:1406-1409

61. Huston J, Nicho s DA, Luetmer PH, et a . Blinded prospective evaluation of sensitivity of MR angiography to known intracranial aneurysms: importance of aneurysm size. AJNR Am J Neuroradiol 1994;15:1607-1614

Address of Correspondence: Dr. Dilli Ram Kafle, Institute of Medicine,Maharajgunj

Kathmandu, Nepal; e-mail:kafledilli@yahoo.com 